\title{
Norois
}

Environnement, aménagement, société

$210 \mid 2009 / 1$

L'agriculture et les filières agro-alimentaires en France et en Argentine

\section{Qualité des produits et maîtrise des marchés : le cas des fromages et des AOC jurassiennes}

Markets in agro-food productions: from standards of productions to labels for cheeses of Jura

\section{Daniel Ricard}

\section{(2) OpenEdition}

Journals

\section{Édition électronique}

URL : https://journals.openedition.org/norois/2831

DOI : $10.4000 /$ norois.2831

ISBN : 978-2-7535-1558-1

ISSN : 1760-8546

Éditeur

Presses universitaires de Rennes

Édition imprimée

Date de publication : 1 mars 2009

Pagination : $91-105$

ISBN : 978-2-7535-0844-6

ISSN : 0029-182X

Référence électronique

Daniel Ricard, «Qualité des produits et maîtrise des marchés : le cas des fromages et des AOC

jurassiennes », Norois [En ligne], 210 | 2009/1, mis en ligne le 15 juin 2009, consulté le 14 janvier 2022.

URL : http://journals.openedition.org/norois/2831; DOI : https://doi.org/10.4000/norois.2831

Ce document a été généré automatiquement le 14 janvier 2022.

(c) Tous droits réservés 


\section{Qualité des produits et maîtrise des marchés : le cas des fromages et des AOC jurassiennes}

Markets in agro-food productions: from standards of productions to labels for cheeses of Jura

Daniel Ricard

\section{NOTE DE L'ÉDITEUR}

Cet article a été reçu le 22 juin 2008 et définitivement accepté le 20 février 2009.

1 Pour de nombreux géographes ou économistes, tels B. Pecqueur (2006), la niche des produits de qualité est particulièrement opportune car elle permet d'échapper en partie à la concurrence, cette dernière se déplaçant alors des produits vers les territoires. Les exemples sont nombreux qui confortent cette thèse : pensons au fromage de Roquefort, à l'huile d'olive de Nyons ou encore aux grands crus bourguignons. Il n'en demeure pas moins que la concurrence règne sans détours à l'intérieur de ces filières haut de gamme, entre les différents opérateurs du marché. Cela n'empêche pas, toutefois, ce créneau spécifique d'apparaître comme une opportunité de choix pour nombre de producteurs et de transformateurs : investir dans la qualité, c'est espérer mieux valoriser sa matière première, son savoir-faire, parfois son terroir.

2 De telles opportunités attirent alors nécessairement les acteurs économiques, au point que ces filières de qualité peuvent être rapidement confrontées à un nouvel écueil : comment éviter la surproduction qui menacerait la performance économique et serait inévitablement préjudiciable pour tous? Ce risque de déséquilibre du marché pose la délicate question de la gestion de l'offre dans un univers fondamentalement concurrentiel. 
3 La plupart des " grandes " productions agricoles européennes s'appuient sur diverses stratégies qui, finalement, mêlent loi du marché et intervention publique. De leur côté, et pour des raisons structurelles (diversité des situations, poids économique souvent limité, faiblesse du lobbying également...), les produits de qualité ne bénéficient pas d'instruments communautaires spécifiques de gestion des marchés comme il en existe par exemple pour le blé ou le lait. Ils ont alors souvent tendance à développer des structures interprofessionnelles, collectives, dont la capacité d'intervention est réelle et à s'appuyer sur des cahiers des charges, souvent contraignants, qui s'imposent à tous. Ces modes d'organisation ne sont pas sans effet sur la gestion de ces « marchés de la qualité ".

4 Après être rapidement revenu sur le traitement général de la filière laitière au sein de la Politique Agricole Commune (PAC), et notamment sur les mécanismes spécifiques qui permettent de réguler ces marchés, cet article abordera plus particulièrement le cas des filières fromagères jurassiennes. Celles-ci, historiquement très structurées et économiquement performantes, attirent mécaniquement de nouveaux intervenants à la recherche d'une meilleure valorisation du lait avec à la clef un risque évident de déstabilisation des marchés, ce qui n'a pas manqué de susciter des stratégies collectives de maitrise de l'offre.

\section{La gestion des marchés: des produits laitiers en général aux produits de qualité en particulier}

5 Quelques remarques préalables s'imposent ici, semble-t-il, afin de mieux replacer les productions fromagères de qualité au regard des grands mécanismes de fonctionnement des marchés agricoles communautaires (Fumey, 1997). On sait en effet que la PAC fonctionne selon des modalités spécifiques, associant loi du marché et concurrence d'une part, et intervention publique (communautaire) d'autre part. Le monde laitier en offre une bonne illustration. La concurrence règne en maître dans cette filière, mais cette dernière s'appuie aussi, depuis 1962, sur des mécanismes d'interventionrelatifs aux marchés du beurre et de la poudre de lait écrémé, associés à la prise en charge d'un système de stockage communautaire par les pouvoirs publics. Cette Organisation Commune des Marchés (OCM-Lait) a, de plus, été fortement renforcée par la mise en place de quotas de production à compter du $1^{\text {er }}$ avril 1984. La profession laitière est donc très encadrée et apparaît ainsi à l'abri des grands soubresauts du marché. En 1986, alors que la CEE croulaitsous plus de deux millions de tonnes de stocks de beurre et de poudre de lait, la matière grasse laitière conservait une certaine valeur, à l'évidence totalement déconnectée du marché.

6 La tendance récente est toutefois manifestement à une plus grande prise en compte des réalités du marché (Boussard et al., 2005). La Commission Européenne ne s'en cache pas et les différentes réformes de la PAC, passées ou à venir, vont en ce sens. L'évolution récente du marché des produits laitiers en est la plus parfaite illustration. Fin 2006début 2007, des déséquilibres naissent sur les marchés internationaux, qui stimulent une fantastique progression des cours. La poudre de lait écrémée passe ainsi de $2400 € /$ tonneà $4000 €$ entre février et juin 2007 ! Ces excès du marché se répercutent assez rapidement sur le prix du lait qui augmente, tant chez le producteur que chez le consommateur. Mais, en quelques mois, ce qu'il faut bien appeler une "bulle spéculative » se dégonfle et l'on revient à $2400 € /$ tonne dès la fin de l'année. Une telle 
situation conduit d'ailleurs à des relations conflictuelles au sein de la filière, le prix du lait apparaissant inexorablement orienté à la baisse dès l'été 2008 .

7 Le poids du marché a donc manifestement beaucoup évolué depuis 1986 ! C'est toutefois oublier que les filières laitières demeurent encadrées par des politiques publiques toujours bien présentes. Les quotas notamment, régulent rigoureusement la collecte (et donc la production) de lait depuis bientôt 25 ans, et les mécanismes de l'Intervention jouent toujours, même s'ils sont beaucoup moins attractifs qu'il y a vingt ans.

8 Venons-en maintenant aux filières de qualité. Les productions haut de gamme bénéficient, elles aussi, de la PAC. Ainsi, les fromages d'AOC profitent bien des mécanismes de l'OCM-Lait, et notamment de la régulation exercée par les quotas. Constatons toutefois que ce sont bien dans les plaines que se concentrent les beurreries et les tours de séchage qui sont, depuis 1962, au cœur de l'Intervention communautaire. Les filières laitières de qualité, en revanche, produisent essentiellement des fromages qui sont exclus de ce même dispositif. Certes, les fromageries du Jura ou de la Savoie peuvent " dégager » du lait vers les tours de séchage mais, en l'absence d'outils de transformation sur place, elles le font alors dans des conditions qui leur sont rarement favorables : actions ponctuelles, obligation de passer par un intermédiaire, absence totale de contrôle de l'aval...

9 Les productions de qualité doivent donc s'imposer d'elles-mêmes. Cet apparent paradoxe s'explique, au moins en partie. Aux yeux du législateur, ces spécialités permettent en effet de dégager une rentabilité satisfaisante sur le marché à la faveur de leurs qualités intrinsèques et n'ont donc pas à être soutenues par des aides qualifiées d'ailleurs par Bruxelles de compensatrices. Imaginerait-on des aides PAC versées aux producteurs de vin de Champagne ou des DPU octroyés aux viticulteurs des grands crus bourguignons ?... À l'inverse, la question de la reconnaissance financière des fonctions non productives associées à ce type de productions reste entière : ne serait-il pas également cohérent de rémunérer l'entretien d'un paysage, voire la préservation d'un patrimoine ? De même doit-on s'interroger à propos des démarches qualitatives émergentes. Encore confidentielles, structurellement peu rentables parce qu'incapables de réaliser des économies d'échelle, elles apparaissent livrées à elles-mêmes et connaissent bien souvent des débuts difficiles. Le soutien des collectivités locales ou des Chambres d'Agriculture leur est alors souvent indispensable. Dans un cas comme dans l'autre, on rejoint les problématiques relevant du second pilier de la PAC. Les productions de qualité sont donc, à l'évidence, nettement aux prises avec le marché, plus nettement peut-être que certaines productions de masse, qui peuvent toujours recourir, si besoin, au débouché qui représente l'Intervention. C'est certainement pour elles une faiblesse structurelle. Ne s'agit-il pas aussi d'un atout puisque, en relation permanente avec le consommateur, elles sont a priori plus réactives, bref, d'une certaine manière, plus autonomes et peutêtre mieux armées, plus solides...

10 Cette situation particulière conduit à s'interroger sur le fonctionnement de ces marchés de la qualité et sur la recherche de l'équilibre entre l'offre et la demande, un équilibre bien entendu déterminant. La concurrence classique entre les intervenants y est en général particulièrement développée, mais le poids de la dimension collective de la gestion de telles productions change en partie la donne, avec notamment des syndicats interprofessionnels qui disposent de plusieurs leviers pour agir. 


\section{Cahier des charges}

11 Le cahier des charges - généralement ici le décret d'AOC - est un de ces leviers. Son objectif premier est clairement de garantir au consommateur un certain niveau qualitatif, grâce au respect de conditions de production spécifiques, plus ou moins contraignantes. La récente réforme de l'INAO en a d'ailleurs renforcé le contrôle, désormais confié à des organismes certificateurs indépendants. Mais le rôle de ce même cahier des charges n'est pas neutre dans la maîtrise des marchés, dans la gestion de l'offre. Intégrer une démarche de qualité exige en effet de respecter des conditions de production rigoureuses. Ainsi, afin de produire du comté ou du reblochon, faudra-t-il supprimer l'ensilage et, pour intégrer la filière laguiole, devra-t-on abandonner la race Holstein. De même, pour bénéficier de l'appellation d'origine, le producteur de saintnectaire devra-t-il fabriquer deux fois par jour et, pour faire du morbier AOC, il conviendra de renoncer à tout pasteurisateur... Tout cela exige un certain engagement personnel, représente un coût d'adaptation et s'accorde mal, de fait, avec une sorte de " zapping » qui se traduirait par des allers et retours incessants entre l'AOC et le produit standard, au gré des seules opportunités économiques. Bref, les transferts de matière première vers les filières d'AOC sont rendus difficiles par les exigences formulées dans le cahier des charges, celui-ci agissant également comme un système de gestion de l'offre qui s'avère d'ailleurs assez efficace. L'agriculture biologique s'appuie sur une protection de nature assez semblable, par le biais cette fois d'un cahier des charges particulièrement rigoureux, à tel point d'ailleurs que certains considèrent que ce dernier constitue un frein au développement des produits bio !

Le « zapping " s'avère en revanche nettement plus aisé dans le cas de produits labellisés reposant sur des cahiers des charges a minima. Évoquons là nombre d'anciens Certificats de Conformité, comme d'ailleurs la plupart des AOC fromagères auvergnates. L'adhésion à une filière de qualité y apparaît bien plus facile, la principale contrainte étant d'être situé dans la zone d'appellation... L'éleveur laitier des monts d'Auvergne, de la Châtaigneraie cantalienne ou des Ségalas est d'ailleurs loin de savoir si son lait sera dirigé vers une fabrication labellisée ! Le « zapping » devient même souvent la règle au sein de ces filières mal encadrées et la gestion des fabrications se raisonne globalement, à l'échelle de l'entreprise, souvent de grande taille, sans incidences sur le paiement de la matière première puisque le lait destiné à l'AOC ne bénéficie généralement pas de prime spécifique ! L'avantage financier est faible, voire nul pour le producteur, ce qui change la donne et relativise beaucoup l'intérêt de ce «zapping ».

\section{Aire d'appellation d'origine contrôlée}

13 L'existence d'une aire d'AOC (ou d'IGP) au sein de laquelle il y a " exclusivité de l'usage du nom » peut apparaître comme un autre instrument de gestion de l'offre. On aborde ici la question majeure du monopole géographique, et surtout de sa justification. Constatons que l'Union Européenne, manifestement plutôt encline au libéralisme qu'au dirigisme, a pourtant entériné, dès 1992, le principe de telles aires exclusives en reconnaissant les IGP et les AOP : c'est là un signe fort. La Suisse fit de même quelques années plus tard. C'est que le monopole géographique paraît recevable dès lors que l'aire exclusive a été délimitée de manière rigoureuse, grâce à un travail scientifique incontestable. De nombreux chercheurs se sont d'ailleurs emparés de cette question, tels ceux du groupe de travail pluridisciplinaire réuni autour de Claude Béranger sous l'autorité de l'INRA 
et de l'INAO, ce dernier Institut ayant organisé en 2000 en Arles un important colloque sur ce thème. On sait aussi que les géographes ruralistes sont nombreux à publier sur ce sujet. Sur le terrain, les experts de l'INAO délimitent les zones d'appellation à la lumière de toute une série de critères relevant des sciences humaines comme de sciences plus « dures ", selon une méthodologie qui, à l'évidence, s'est nettement améliorée depuis 1990. Les travaux s'étalent désormais sur plusieurs années et les rapports d'expertise atteignent souvent la centaine de pages. C'est dans cet esprit que les zones d'AOC du salers, du roquefort et de l'ancienne fourme d'Ambert ou de Montbrison ont été revues et que les dossiers du camembert de Normandie, du bleu d'Auvergne et du picodon devraient aboutir prochainement. Et, si le fromage de cantal s'est toujours montré réticent, le comté engagea, de lui-même, dès le début des années 1990, la refonte de son aire géographique (fig. 1).

Figure 1 : Le massif jurassien, carte de localisation et évolution de la production de comté (INAO, 2007) Location map of Jura Mountain and production trends of Comté, Jura's guaranteed of quality cheese

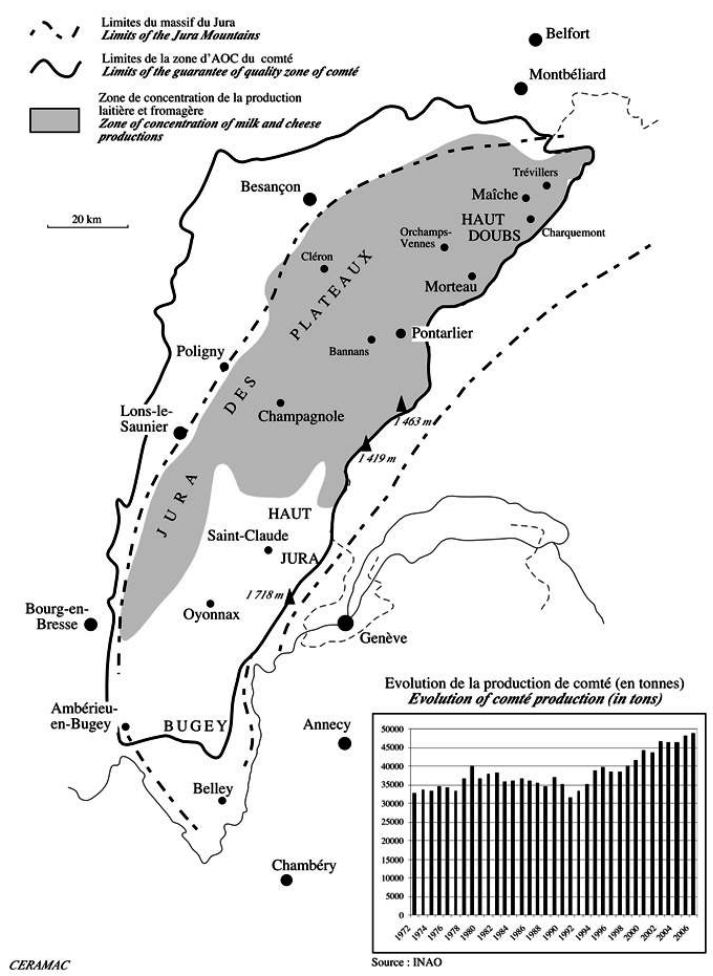

La révision d'une aire d'AOC vise d'abord à délimiter un terroir plus cohérent et apparaît donc, avant tout, comme une forme de renforcement de l'ancrage territorial d'un fromage, d'un vin ou d'une huile et, au final, de sa qualité spécifique. Constitue-telle également un mécanisme déguisé de maîtrise de l'offre ? La réponse est complexe. Signalons d'abord que les syndicats hésitent souvent à ouvrir de tels dossiers et qu'ils cherchent manifestement, lorsque tel est le cas, à préserver le potentiel productif le plus significatif. Et que dire de la zone d'AOC de l'abondance, démesurément étendue lors de sa délimitation en 1990, au simple motif que ce fromage devait disposer d'un potentiel de développement suffisant ! On est loin de toute attitude protectionniste ! En revanche, la plupart des révisions conduisent bien à une restriction de l'aire délimitée. La zone de production du lait destiné au roquefort, fut ainsi réduite des trois-quarts en 
2001 ! Quant au terroir du comté, il fut amputé de l'ensemble du département de la Haute-Saône en 1998. Mais le Comité Interprofessionnel du Gruyère de Comté (CIGC) s'est toujours défendu de toute visée protectionniste, arguant du fait que la nouvelle zone offrait un potentiel de l'ordre du double de la production actuelle de comté. Et dans le cas du roquefort, l'ampleur de la réduction doit être miseen relation avec une délimitation antérieure totalement dépassée. Ni en Franche-Comté, ni dans l'Aveyron, la maitrise de l'offre ne semble donc avoir été décisive dans le processus de révision/ réduction de la zone de production. À l'inverse, reconnaissons qu'une aire d'appellation de taille réduite, n'autorisant qu'une production fromagère limitée, apparaît bien comme un atout. La gestion de l'offre y devient alors plus aisée, d'autant qu'elle se combine avec l'action régulatrice des quotas laitiers. Tel est parfaitement le cas de la zone du beaufort, où la totalité du lait est désormais transformée en AOC. Le livarot, le laguiole, voire le bleu de Gex, le reblochon et le mont d'or bénéficient aussi d'une telle forme de régulation. S'agissait-il, à l'époque, d'une volonté affirmée de repli protectionniste? A priori non. La zone du beaufort doit sa situation actuelle aux difficultés structurelles de la collecte dans une montagne difficile. Quant à l'aire du livarot, rigoureusement limitée à l'époque, on cherche plutôt à l'élargir aujourd'hui.

\section{Organisations interprofessionnelles}

Dernier levier important de gestion de l'offre au service des productions fromagères de qualité : le recours à des organisations interprofessionnelles fortement structurées. Cette attitude vaut d'ailleurs pour l'ensemble d'une filière laitière très organisée. Ici, les fédérations professionnelles représentatives (FNPL, FNIL, FNCL, ATLA) sont puissantes, au point notamment de négocier collectivement le prix du lait, même si ce dispositif est actuellement remis en cause. À la clef, le producteur français bénéficie d'une sorte de prix de référence, grossièrement équivalent d'une région à l'autre et d'une entreprise à l'autre, quasi garanti et relativement stable, au moins jusqu'à ces derniers mois. Une telle dimension collective se retrouve en Europe du Nord (Allemagne, Pays-Bas, Danemark), bref là où la production est la plus importante et souvent avec l'appui d'une coopération omniprésente. Même la très libérale Grande-Bretagne fut historiquement dominée par le Milk Marketting Board, acheteur unique du lait pour tout le pays et dont le monopole ne fut démantelé que dans les années 1990. Des interprofessions très organisées se rencontrent également en Slovaquie.

Les observateurs conviennent que le caractère spécifique de la production laitière explique de tels comportements. Produire du lait exige de lourds investissements et donc un horizon relativement dégagé, d'autant que la production ne peut être suspendue, même une journée. Quant aux transformateurs, souvent confrontés à des produits difficiles à stocker, ils doivent produire en continu. Tout cela favorise les partenariats de moyen ou de long terme.

17 Cette inclinaison à la régulation des apports se retrouve dans les productions de qualité, mais avec certaines spécificités. Ces filières échappent en effet pour une bonne partie aux régulations interprofessionnelles nationales précédemment décrites et sont beaucoup plus directement en prise avec leurs marchés, surtout dans les «montagnes fromagères ". Cela n'a toutefois pas empêché la mise en place de systèmes assez élaborés de gestion des volumes et du marché, comme on va le voir. 

transformateurs, gère depuis 1988 et en l'absence de quotas laitiers dans le secteur du lait de brebis, un système de références individuelles attribuées aux éleveurs et qui s'accompagne d'une forme de partage du marché entre les transformateurs (Rieutort, 1995). Une grille de paiement, négociée annuellement, s'applique à l'ensemble de la collecte. Le lait destiné au roquefort est très bien rémunéré, alors que celui qui est orienté vers les produits de diversification, puis de dégagement, doit se contenter de prix nettement plus faibles'. Dans une région où l'AOC ne valorise que 60 à $70 \%$ de la collecte, la stratégie s'avère payante. Les éleveurs peuvent produire plus, certes à des prix plus faibles, mais surtout sans déstabiliser le marché du roquefort. Un tel système apparait en revanche très sélectif : il privilégie les acteurs les plus performants, consolide les parts de marché des fromageries et conforte les producteurs anciens, qui bénéficient à plein de ces références « historiques ».

Le département du Cantal expérimenta d'autres voies. Le Comité Interprofessionnel des Fromages mit en effet en place, en 1976, une caisse de péréquation destinée à aider les entreprises orientées vers les produits de dégagement, au moyen d'un prélèvement uniforme opéré sur les fabrications de cantal AOC (Ricard, 1994). En 1987, le CIF différencie le taux de cotisation : celui-ci sera de $0,88 \mathrm{~F} / \mathrm{kg}$ pour les fabrications de cantal correspondant aux 39 premiers pour cent de la collecte des laiteries lors de l'année précédente, puis de $1,32 \mathrm{~F} / \mathrm{kg}$ pour la tranche comprise entre 39 et $100 \%$ de la collecte, et enfin de $2,64 \mathrm{~F} / \mathrm{kg}$ pour le lait transformé en cantal et qui dépasserait $100 \%$ de la collecte de l'année précédente. Ce mécanisme fort complexe visait à éviter un accroissement des fabrications dans un marché du cantal souvent saturé. Il se révéla en réalité fort discriminatoire. D'une part, les entreprises diversifiées, relevant essentiellement de la seule tranche A, étaient nettement moins sollicitées tout en encaissant l'ensemble des aides collectées par la caisse de péréquation. D'autre part, les ateliers monovalents payaient au prix fort, ne bénéficiaient pas des aides et étaient les seuls à devoir payer $2,64 \mathrm{~F} / \mathrm{kg}$ s'ils voulaient augmenter leur production!

Les deux stratégies sont a priori orientées vers le même objectif, à savoir gérer le marché, mais les stratégies et les résultats obtenus s'avèrent inégaux. À Roquefort, on cherche à valoriser le mieux possible le lait destiné à l'AOC, quitte à s'appuyer sur un dispositif finalement très sélectif. Le Cantal lui, adopte une politique nettement plus égalitariste, soutenue par le syndicalisme agricole et la coopération : on transfère les plus values issues des AOC et des entreprises artisanales vers les pâtes italiennes, le cheddar et les grands groupes... Quelques années plus tard, la justice reconnaîtra la non-conformité de ce mécanisme vis-à-vis des règles de la concurrence et cassera le « Plan cantal».

\section{Un cas concret d'optimisation de la gestion des filières de qualité : le massif jurassien}

21 Dans la chaîne jurassienne (fig. 1), les mécanismes de gestion des marchés apparaissent nettement plus élaborés et constituent, pour notre réflexion, un exemple régional fort instructif (Callois et al., 2009). Ici en effet, on s'appuie sur une vieille tradition de partenariat et de négociation, ainsi que de partage de la valeur ajoutée entre 
producteurs, fromageries et affineurs, le tout passant par une gestion véritablement collective des marchés fromagers.

\section{Quelques données de cadrage}

Dans le Jura, la production laitière est omniprésente, au moins au nord d'une ligne Oyonnax/Saint-Claude (fig. 1). Autre tradition, celle d'une transformation fromagère associée à la fruitière villageoise, avec à la clef un fort ancrage territorial. S'y ajoute une excellente valorisation de la matière première, le prix du lait y étant structurellement plus élevé que la moyenne nationale (fig. 2).

Toutefois, le massif est loin d'être homogène. Ainsi, tout le lait n'est pas destiné aux filières d'appellation, soit que les fromageries aient choisi d'autres stratégies (telles Perrin-Vermot à Cléron), soit que les éleveurs aient privilégié un modèle plus intensif (vaches Holstein, ensilage d'herbe ou de maïs), se maintenant alors en dehors du monde des AOC. En outre, les fromages jurassiens offrent des performances assez inégales. Le comté fait référence avec près de $50000 \mathrm{t} / \mathrm{an}$ (fig. 1) et un prix du lait voisin de $0,35 € / \mathrm{l}$. Le bleu de Gex offre une rentabilité similaire, mais pour un tout petit marché. Le mont d'or apparait le plus performant (de l'ordre de $0,40 € / l$ ) mais ne se fabrique qu'en hiver. Quant à l'emmental Grand Cru, vigoureusement concurrencé sur le marché des pâtes dures à ouvertures, il ne génère aujourd'hui que peu de valeur ajoutée, en dépit de sérieuses contraintes. Enfin, l'accès à l'AOC a dynamisé cet autre produit traditionnel qu'est le morbier, tout en le recentrant sur sa montagne originelle. 
Figure 2 : Le prix du lait en France et dans les filières d'AOC Milk price in France and in guarantee of quality productions

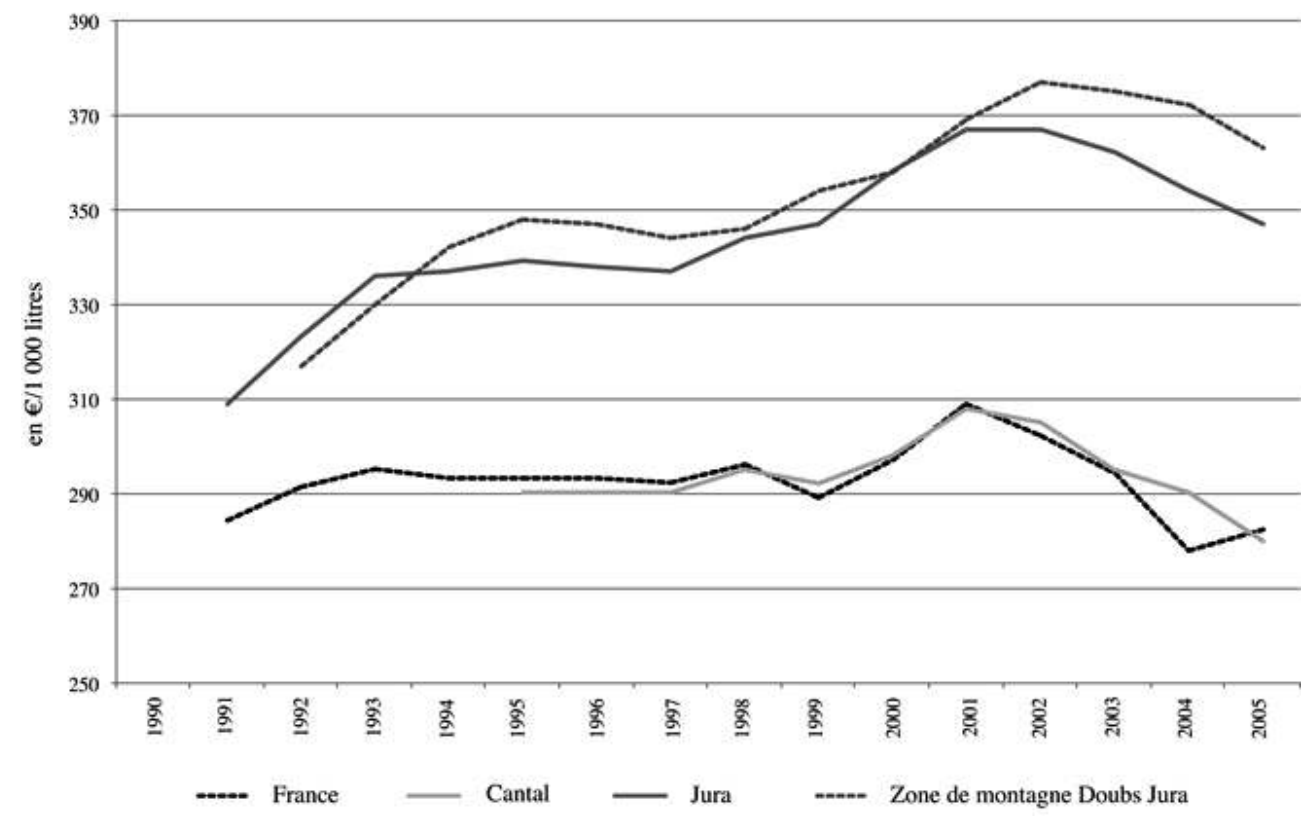

Les statistiques relatives au prix du lait dans les filières fromagères d'AOC sont, pour tout dire, inexistantes. Ce graphique prend en compte plusieurs séries statistiques. La courbe relative au prix du lait en France fera ici référence. Dans le département du Cantal, les prix ne sont guère différents selon la destination de la matière première. Cette donnée illustre un contexte régional marqué par les fromages d'appellation mais où l'on n'a guère cherché, justement, à maîtriser ni à différencier l'offre spécifique d'AOC. Le prix « Doubs-Jura » concerne deux départements où les produits labellisés absorbent 80 à $85 \%$ de la collecte. Enfin, la courbe relative aux seules zones de montagne de ces deux départements est celle qui permet d'approcher au plus près la valorisation réellement procurée par les seuls fromages d'AOC, puisque le taux d'utilisation de la collecte se rapproche ici des $90 \%$. D'après services statistiques, CNIEL et P. Jeanneaux (2009).

En revanche, s'il est un terrain où le Jura apparait fort homogène, c'est bien celui des stratégies fromagères. Ici, la gestion des AOC s'avère toujours rigoureuse, fondée sur des cahiers des charges exigeants, que l'on soit en comté, en mont d'or, en bleu de Gex, en morbier, tout comme en emmental Grand Cru et, aujourd'hui, en gruyère. Il s'agit là de stratégies de long terme, qui ont stimulé une nette croissance des volumes fabriqués, à l'exception notable de l'emmental labellisé, voire du confidentiel bleu de Gex. Le mont d'or est passé de quelques centaines de tonnes dans les années 1980 à plus de $4000 \mathrm{t} / \mathrm{an}$ actuellement. Le morbier, jadis concurrencé par des produits venus de Bretagne, des plaines de la Saône ou du Livradois, a aussi beaucoup progressé : le massif en produisait 2700 tonnes lors de l'accès à l'AOC en 2000 contre 8200 tonnes en 2007 ! Quant au comté, il n'a cessé de conquérir de nouveaux marchés, passant de 30000 tonnes vers 1980 à 40000 tonnes dans les années 1990 pour friser aujourd'hui les 50000 tonnes (fig. 1).

Toutes ces dynamiques positives contrastent avec les difficultés rencontrées par les produits laitiers industriels (beurre, poudre), par le lait UHT, voire par les fromages de grande consommation.

\section{Des filières de qualité attractives}

Venons-en à la question de l'accès à ces filières attractives. Dans ce contexte jurassien, le passage d'une $\mathrm{AOC}$ à l'autre ne pose pas de réelles difficultés à l'éleveur, dès lors qu'il 
est localisé dans des aires délimitées qui se superposent, compte tenu de la relative homogénéité des différents cahiers des charges. L'obstacle est à l'évidence ailleurs, on l'a dit plus haut, quand il s'agit de quitter la production « standard » pour livrer son lait à une fromagerie spécialisée en AOC. Au stade de la transformation, les conversions sont en revanche toujours coûteuses, compte tenu des spécificités liées aux technologies de fabrication. Les fromageries doivent en effet reconsidérer leur outil de production si elles veulent se diversifier ou se réorienter, une obligation toutefois si l'on veut fabriquer du mont d'or, dont l'AOC n'est que saisonnière.

Tous ces obstacles n'ont toutefois pas empêché certains transferts de matière première, différentiel de rentabilité aidant. Certains producteurs de lait ont ainsi choisi, individuellement, d'adapter leurs systèmes de production afin de rejoindre une filière jugée plus rémunératrice. En aval, le mouvement est d'une autre ampleur, avec plusieurs entreprises régionales qui ont abandonné, depuis les années 1980, lait de consommation, produits frais et fromages pasteurisés pour s'intéresser davantage à ces productions plus valorisantes, avec d'ailleurs plus ou moins de réussite.

Le parcours des coopératives de Mont Rivel, près de Champagnole et de Bannans, près de Pontarlier, est riche d'enseignements (Ricard, 1994). Créées vers 1970 dans le cadre de la restructuration des fruitières impulsée par la "Charte du comté ", elles ne parviennent pas à maîtriser les fabrications traditionnelles dans leurs gros ateliers et adoptent l'emmental pasteurisé, avec à la clef une sous-valorisation structurelle de leur collecte. Vers 1990, ces coopératives reviennent vers le lait cru, la première privilégiant le comté, la seconde s'imposant comme le leader de l'appellation mont d'or. Dans le même temps, beaucoup de fruitières villageoises qui maintenaient une certaine diversification, se sont recentrées sur le seul comté, manifestement plus facile à commercialiser. Mais parfois, la réorientation de l'outil de transformation échoue, comme chez Schneiter à Santoche, près de Clerval, incapable de lutter avec les « géants » du lait de consommation, d'autant qu'il faut compter avec un contexte régional de lait cher. La laiterie doit rejoindre la grosse coopérative vosgienne Ermitage, qui se contente alors de redistribuer la matière première vers l'ensemble de ses sites industriels. Même chose pour la Centrale Laitière de Franche Comté, à l'étroit dans une banlieue de Belfort, exclue de toute zone d'AOC et constamment menacée sur le marché de l'emmental pasteurisé. Elle doit son salut à un triple choix stratégique : l'exploitation d'un atelier largement amorti, une alliance avec des coopératives lorraines... et l'entrée récente sur le marché du gruyère. Hélas, exclue de l'aire délimitée de cette nouvelle $\mathrm{AOC}$, cette dernière diversification tourne court. Au total toutefois, les réussites l'emportent sur les échecs et une bonne centaine de millions de litres de lait ont rejoint depuis vingt ans les différentes filières d'AOC dans le massif du Jura, essentiellement en provenance de grosses laiteries orientées jusque-là vers des productions plus banales.

Signalons qu'un autre type de transfert se réalise au sein même du marché des productions labellisées, avec notamment une progression des « petites » appellations et un recul de l'emmental au lait cru. Ce fort développement du mont d'or et du morbier ne pose toutefois pas de problèmes spécifiques sur les marchés : le premier étend son rayonnement commercial en misant sur le caractère très spécifique du produit, quand le second récupère les consommateurs délaissés par les anciens intervenants, progressivement exclus de la filière suite à la mise en place d'une aire d'appellation recentrée sur le seul massif jurassien. 


\section{Le comté et ses "plans de campagne »}

30 campagne ». Ces derniers ouvrent la possibilité aux fabricants de pâtes pressées cuites (comté, emmental, beaufort) de présenter à l'Administration un plan qui prévoit, compte tenu des évolutions techniques et économiques, un programme d'organisation de la production. Le plan de campagne fait le bilan de la situation et propose des mesures qui peuvent aller jusqu'à l'activation de clauses de sauvegarde en cas de problème grave, comme le fit le comté en 1995. Le CIGC, rappelons-le, fixe le prix des plaques d'identification dont il a le monopole de la vente auprès des fromageries et le plan de campagne lui offre la possibilité de vendre ces « plaques vertes » à un tarif plus élevé au-delà d’une certaine référence fixée par rapport à un historique. Chaque année, le CIGC détermine donc pour chaque fromagerie cette référence qui est de l'ordre de 100 à $102 \%$ des fabrications de l'année précédente. La plaque vaut $4,80 €$ en 2008. Les fromageries peuvent produire au-delà de cette référence, mais les plaques vertes sont alors vendues 10, voire 15 et même aujourd'hui, 20 fois le prix de base ! Bref, ce mécanisme, qui s’apparente à un quota par fromagerie, permet de réguler efficacement la production.

\section{La crispation des positions autour du haut Doubs}

Si le comté parvient ainsi à maîtriser globalement son marché, le renforcement des animosités est toutefois évident entre ceux qui bénéficient des plaques vertes et ceux qui n'en ont pas! Tel est bien le cas dans le haut Doubs où de grosses fruitières exploitées en gestion directe ou par des fromagers privés, souvent d'origine suisse, fabriquaient traditionnellement de l'emmental. Dès la fin des années 1960, ces fromageries sont victimes de la concurrence de l'emmental breton et la mise en place d'un Label Rouge (1978), puis d'une IGP (1996), ne parviennent qu'à limiter le recul structurel des ventes.

Ces fruitières cherchent alors à " passer au comté ", ce qui n'est guère du goût du CIGC qui perçoit là un risque de déstabilisation de son propre marché : n'y a-t-il pas ici, au nord de Morteau, plus de cent millions de litres de lait répartis sur soixante communes? Pendant quelques années, le plan de campagne du comté autorise un système d' " ouverture préférentielle ", au profit des candidats à la conversion, en fonction des possibilités offertes par le marché. Les demandes les plus importantes émanent alors justement des fruitières à emmental du haut Doubs. Si les plus petites de ces 
coopératives passent au comté, dès avant les plans de campagne d'ailleurs, les plus grosses, celles d'Orchamps-Vennes, de Trévillers et de Charquemont, ne bénéficieront jamais de plaques vertes en quantité suffisante. Depuis le début des années 2000, la conversion est d'ailleurs au point mort.

À la même époque, ces entreprises, de plus en plus déstabilisées sur leur marché du Grand Cru, trouvent une nouvelle opportunité dans la fabrication du gruyère. La technologie fromagère est voisine de celle de l'emmental et le marché, relancé par quelques entreprises, apparaît assez prometteur. En outre, la dénomination bénéficie d'une certaine notoriété. Nouveau rebondissement là encore avec la mise en place d'une appellation d'origine. Le CIGC adopte une attitude complexe qui tourne à la franche hostilité lorsqu'il se rend compte que le haut Doubs intègrera bien la future zone d'AOC. La région, contiguë à l'aire d'appellation du gruyère suisse bénéficie en effet d'une certaine antériorité dans l'usage du nom. Jadis, les fromagers suisses y produisaient un emmental largement vendu en dehors de la montagne mais qui n'intéressait guère la clientèle locale. Pour satisfaire cette dernière, ils fabriquaient donc une pâte dure moins ouverte, spécifique puisque l'on n'est pas ici dans une terre de comté : le gruyère du haut Doubs vient de là... Le CIGC ne conteste pas ces éléments qui valident, à l'évidence, l'existence d'un terroir. Il insiste, en revanche, sur les risques de déstabilisation du marché du comté liés à ces possibilités de " zapping » entre comté et gruyère dont disposeront, assure-t-il, les fruitières locales. Toutes les voies de recours ayant été utilisées, la zone est finalement entérinée, mais la procédure a clairement posé, encore une fois, la question de la maîtrise de l'accès au marché et de la gestion des volumes au sein des filières de qualité.

\section{Comment concilier qualité, concurrence et maîtrise des marchés?}

\section{La prise en compte des évolutions du marché et de l'OCM-Lait}

À vrai dire, jurassiens - et savoyards - s'intéressent depuis longtemps à ces problématiques d'ensemble, notamment dans la perspective d'un abandon des quotas laitiers par Bruxelles. Pour la Commission Européenne en effet, une telle libéralisation doit permettre de restructurer la profession, de produire plus, de réaliser des économies d'échelle et donc, au final, d'améliorer le revenu des producteurs. Mais, pour les Jurassiens, la rentabilité passe bien plus par le maintien d'un haut niveau qualitatif associé à la promotion d'un terroir fromager et à une certaine maîtrise des volumes. Les points de vue semblent difficilement conciliables. Essayons d'y voir plus clair.

Les Jurassiens avaient engagé une réflexion sur ce sujet dès le milieu des années 1990 et mirent alors sur pied une commission ad hoc. Pour eux en effet, la disparition des quotas ne peut que conduire à une augmentation de la production laitière dans le massif et à une inévitable progression de l'offre de comté. Les risques de surproduction leur apparaissent donc évidents à moyen terme. D'autant que la suppression des quotas se traduira par une volonté accrue de conversion chez les fabricants de produits laitiers standardisés. Bref, on est convaincu qu'il y a péril à un horizon assez rapproché.

La démarche de réduction de l'aire d'AOC du comté doit-elle être rattachée à une stratégie que l'on pourrait alors qualifier de protectionniste ? Certainement en partie, mais là ne semble pas l'essentiel si l'on en croit les conversations de l'époque avec les 
professionnels de la filière : ces derniers soulignent d'ailleurs, et à juste titre nous semble-t-il, que le potentiel de l'actuelle aire d'AOC dépasse encore les 100000 tonnes de comté. Constatons aussi que ce chantier avait été engagé dès le début de la décennie.

Au-delà, les Jurassiens prennent assez rapidement conscience que la Commission Européenne n'acceptera aucune limitation de l'accès direct au marché, ce qui serait contraire à sa philosophie. Dès lors, les plans de campagne, comme tout système de contingentement, s'avèrent juridiquement fragiles ${ }^{2}$. Ajoutons que la loi de " Modernisation de l'Économie ", votée par le Parlement en 2008, va dans le même sens en privilégiant l'amélioration du pouvoir d'achat des consommateurs. Enfin, la disparition des quotas pour 2015 semble désormais actée. Bref, l'heure n'est plus à l'interventionnisme... À l'inverse, ces mêmes Franc-comtois prennent progressivement conscience que Bruxelles est beaucoup plus réceptif aux notions de protection de la qualité, de produits spécifiques et de respect de l'environnement. Reste donc à démontrer que le maintien de la qualité et du patrimoine fromager local passe aussi par le respect de conditions de production strictes, quitte à exiger ce qui pourrait apparaître comme des entraves au libéralisme classique. On s'interroge donc sur la caractérisation du terroir, le rôle du lait cru, l'extensification ou encore la gestion optimale et durable des prairies. On s'interroge aussi sur l'image du produit et la place de la fruitière, ce qui ne manque pas de transparaître dans les campagnes publicitaires télévisuelles. Plusieurs décisions assez révolutionnaires sortent de cette réflexion.

\section{La mise en place de mécanismes de gestion du marché originaux}

Le CIGC propose ainsi de limiter les aires de collecte des fromageries! L'affaire fait grand bruit car elle bloque toute stratégie de restructuration des ateliers avec transport du lait sur de longues distances comme le pratiquent déjà quelques gros intervenants. Lactalis, le plus important groupe laitier français, porte d'ailleurs l'affaire en justice, mais la décision du Conseil d'État ne lui est pas favorable. Au final, cette mesure décisive subit quelques aménagements mais est avalisée dans le nouveau décret signé en 2000. Les producteurs de lait devront être localisés dans un cercle de $25 \mathrm{~km}$ de diamètre, centré ou non sur la laiterie, sans effet rétroactif. La mesure renforce assurément la qualité du comté, considérant qu'un transport sur de trop longues distances maltraite bien la matière première. Mais il s'agit surtout de préserver l'image du produit, en confortant donc la fruitière. L'objectif est là, mais il en résulte aussi que le mécanisme, franchement contraignant, freine l'accès à l'AOC des grands groupes industriels, peu enclins à se soumettre à de telles exigences qu'ils jugent antiéconomiques. On est donc pour partie en face d'une forme d'encadrement de la production, bien que l'accès au marché reste parfaitement libre pour les fromageries. Encore faut-il que ces dernières acceptent des règles collectives qui sont autant de vraie contraintes, susceptibles de décourager des industriels habitués à plus de liberté ainsi que des opportunistes préférant agir sur un horizon de court terme.

D'autres pistes de réflexion sont abordées comme cette proposition novatrice d'une délimitation parcellaire, à l'image de ce qui se pratique dans le vignoble, l'huile d'olive ou les lentilles. On n'envisage pas moins de passer l'immense zone d'appellation du comté au peigne fin afin de qualifier les seules parcelles jugées aptes à la production laitière en fonction de critères qui visent à renforcer le lien au terroir ! La réflexion porte également sur une limitation de la production laitière à l'hectare, partant du 
principe que le respect du terroir et de la qualité est incompatible avec toute forme d'intensification jugée excessive. Il convient donc de privilégier l'herbe, surtout la prairie naturelle, quitte à prendre certaines mesures contraignantes. Le syndicat du beaufort avait exploré cette voie en limitant, dès 1993, la production laitière moyenne des exploitations à $5000 \mathrm{~kg}$ de lait par vache et par lactation. Plusieurs interlocuteurs de la grande industrie nous avouaient alors leur grande perplexité vis à vis d'un tel « non-sens économique " qui allait " à l'encontre de toute recherche de l'amélioration des performances, bref de toute idée de progrès ». En réalité, les savoyards se plaçaient déjà dans un autre registre, celui d'une valorisation maximale conditionnée par un respect rigoureux du terroir.

41 Dans le massif jurassien, les différents syndicats d'AOC vont mettre en place des mécanismes similaires de limitation de l'intensification. Le CIGC exige ainsi, dès 1998, un hectare de surface en herbe par vache laitière, avant d'aller plus loin en 2007 : il faudra désormais produire moins de 4600 litres/ha de surface fourragère principale (SFP) ${ }^{3}$, avoir moins de 1,3 UGB/ha de SFP et disposer d'au moins un hectare de SFP par vache laitière! L'actuel décret du mont d'or exige également un hectare de SFP par vache laitière, de même que le décret de 2005 relatif au bleu de Gex. Enfin, pour produire du gruyère, faut-il respecter une triple contrainte : moins de 1,4 UGB/ha, moins de $1800 \mathrm{~kg}$ d'aliment concentré par vache et moins de 5000 litres par hectare de SFP. Le mouvement est donc général et ces entraves évidentes à l'intensification et à l'industrialisation classiques sont d'ailleurs validées par le législateur, tant à Paris qu'à Bruxelles.

42 Les réflexions conduites dans l'Est Central sont incontestablement spectaculaires et font rupture avec la pensée économique générale, pour des produits qui, dans le même temps, sont parfaitement en prise avec le marché. On assiste en fait à une forme de refus de la réduction systématique des coûts de production de la part de professionnels qui cherchent, à l'inverse, à protéger un terroir et des savoir-faire, donc la qualité, seule façon, selon eux, de préserver les prix de vente et donc les revenus. Constatons que cette stratégie économique, plutôt à contre courant, porte ses fruits depuis des décennies : le prix du lait reste structurellement élevé, la réduction du nombre des exploitations est plus limitée qu'ailleurs et le volume d'emploi en fromagerie et dans les caves d'affinage demeure bien plus important que dans les filières classiques, toutes preuves incontestables d'un vrai développement local.

\section{L'appui de structures interprofessionnelles puissantes}

43 Avouons que de telles stratégies ne peuvent se comprendre sans prendre en considération cette dimension collective forte qui caractérise les filières fromagères jurassiennes - et savoyardes - et qui s'appuie sur des héritages historiques majeurs (la fruitière, la pratique de l'affouage, les terres collectives...). Sur ce plan, l'Est Central dispose d'organisations professionnelles très structurées et par ailleurs non liées au syndicalisme agricole classique. Tel est le cas des Fédérations Départementales de Coopératives Laitières (FDCL), particulièrement puissantes et dynamiques ici. Les syndicats d'appellation d'origine, devenus aujourd'hui des « Organismes de Défense et de Gestion " (ODG) occupent dans ce contexte une place décisive, notamment dans le cas du comté, géré par un Comité Interprofessionnel à l'autorité bien établie et qui dispose de gros moyens financiers : le CIGC. Tous ces ODG sont en outre réunis, depuis la fin des années 1990, au sein d'une Fédération Nationale des AOC (FNAOC) qui 
rassemble à ce jour 45 \% de la production fromagère française labellisée et est ainsi devenue une organisation qui compte dans le monde des appellations.

Cette réalité jurassienne n'interdit ni les rivalités, ni les conflits, surtout d'ailleurs quand le prix du lait " standard " augmente, comme en 2007, mais la cohésion d'ensemble des acteurs reste claire, pour des filières toujours tenues et contrôlées par les agriculteurs ${ }^{4}$. On est là loin des réalités du Massif central où beaucoup de ces organismes collectifs se sont délitées ou sont désormais contrôlés par de puissants groupes laitiers, coopératifs ou privés, locaux ou non. Il y a quelques années, le CIF, qui gère les destinées du cantal et du salers n'avait-il pas la majorité de ses membres qui résidaient en dehors du département?

\section{Conclusion}

Quel bilan dresser de ces expériences régionales? Constatons que la question de la régulation des marchés se pose bel et bien pour les produits de qualité au sein d'économies de moins en moins régulées. Elle se pose d'autant plus que ces spécialités fromagères de qualité supportent des coûts de productions importants (condition indispensable si l'on veut, justement, conserver cette qualité spécifique) et apparaissent donc, a priori, comme non concurrentielles.

Mais, dans le même temps, des prix de vente élevés rendent ces mêmes produits d'appellation particulièrement attractifs pour des acteurs économiques extérieurs à la recherche de débouchés rémunérateurs. Dès lors, les risques sont réels d'une déstabilisation de ces marchés particuliers.

Tel est particulièrement le cas du Jura et des Alpes du Nord où ce contexte spécifique a conduit les professionnels, et en premier lieu les producteurs de lait, à s'interroger depuis longtemps à propos de l'encadrement et de la gestion de la production laitière et de la fabrication fromagère (Jeanneaux, 2009). Ici, les syndicats d'appellation se sont appuyés sur le contenu des cahiers des charges des différents fromages et ont progressivement renforcé les contraintes relatives aux conditions de production du lait et du fromage dans le cadre d'une démarche visant avant tout à consolider la qualité spécifique des produits d'AOC. Mais de telles exigences limitent, de fait, l'accès au marché, puisque tous les acteurs économiques ne sont pas susceptibles de s'adapter à ces contraintes réglementaires. La réflexion sur la maîtrise des volumes n'est donc jamais très éloignée des considérations relatives à l'expression de la qualité spécifique des fromages.

Reste à savoir si de telles stratégies, à l'évidence efficaces ces dernières années, pourront perdurer si le mécanisme des quotas de production en vigueur depuis 1984 est abandonné, comme cela semble devoir être le cas après 2015. La question est d'importance, puisqu'elle conditionne pour partie la permanence même de ces productions fromagères de qualité dans des marchés toujours plus ouverts et au sein desquels les prix seront nécessairement tirés vers le bas. On est là dans l'arbitrage entre liberté d'entreprendre et protection, pour des produits particuliers qui mettent en jeu des logiques économiques assez spécifiques, marquées par la recherche d'un prix de vente élevé et par une réflexion sur le temps long. 


\section{BIBLIOGRAPHIE}

BOUSSARD (J.-M.), GERARD (F.), PIKETTY (M.-G.), 2005. - « Libéraliser l'agriculture mondiale ? Théories, modèles et réalités ", Montpellier, CIRAD, $136 \mathrm{p}$.

CALlois (J.-M.), JeAnNEAuX (P.), WouTs (C.), 2009. - « Durabilité d'un compromis territorial dans un contexte de pression compétitive accrue : le cas de la filière AOC comté », RERU, sous presse.

Fumey (G.), 1997. - « L'agriculture dans la nouvelle économie mondiale », Paris, PUF, 380 p.

INAO. - Liste des décrets d'AOC [http://www.inao.gouv.fr].

JEANNEAUX (P.), 2009. - Les conditions de pérennité des élevages laitiers de montagne : construire et protéger collectivement les ressources spécifiques des systèmes productifs fromagers, Actes des Journées Rurales de Besançon, sous presse.

PECQUeUR (B.), 2006. -Le tournant territorial de l'économie globale, Espaces et Sociétés, vol. 124-125, $\mathrm{n}^{\circ} 2-3, \mathrm{p} \cdot 17-32$.

RICARD (D.), 1994. - « Les montagnes fromagères françaises », Clermont-Ferrand, CERAMAC, 496 p.

RIEUTORT (L.), 1995. - « L'élevage ovin en France », Clermont-Ferrand, CERAMAC, 512 p.

\section{NOTES}

1. En $2008,88,4$ millions de litres ont été valorisés en roquefort et payés $1,10608 € /$ litre aux producteurs ; 61 millions l'ont été en produits « valorisants » (classe II) à 0,74011 €/litre. Les 11,5 millions de litres orientés vers les produits de dégagement n'ont été réglés que $0,36 € /$ litre (source : Confédération de Roquefort).

2. Le groupe Lactalis (ex-Besnier) n'hésitera d'ailleurs pas à traduire le CIGC en justice, pour un jugement finalement nuancé, favorable sur la forme, mais qui ne condamne toutefois pas le mécanisme des plans de campagne.

3. Selon le Président du CIGC, la moyenne de la filière ressort à 2700 litres par hectare et seulement $5 \%$ des exploitations dépassant 4800 litres.

4. La difficile gestion des stocks de comté est un bon révélateur de ces comportements collectifs. Ces immobilisations, d'autant plus importantes que la durée d'affinage a augmenté significativement ces dernières années, sont traditionnellement supportées conjointement par les affineurs et les fromageries, ces dernières n'étant payées qu'avec un net décalage par rapport aux livraisons. À partir du début du siècle, les stocks s'accumulent et, en 2006, face à une situation jugée alarmante, la profession décide d'un plan de dégagement du marché. Il faut orienter la collecte, durant plusieurs jours, vers des produits de dégagement, peu rémunérateurs, moyennant un soutien financier partiel du CIGC. Quelques mois plus tard, le plan de dégagement, fondé sur le seul volontariat, est réalisé à $80 \%$ environ : les analyses de long terme l'ont manifestement emporté sur les objectifs à courte vue. 


\section{RÉSUMÉS}

Les productions agroalimentaires approvisionnent un commerce soumis à une rude concurrence mais aussi imparfaitement libéralisé (politiques agricoles, barrières douanières...). Dans le cas des productions de qualité, on retrouve cette dualité. Les fromages d'AOC du Jura, et notamment le comté, alimentent ainsi un commerce totalement concurrentiel mais représentent aussi un marché rentable, donc attractif. Il existe alors un vrai risque d'engorgement de ces marchés, par le biais d'entreprises venues de l'extérieur et qui investiraient là, au risque de créer la surproduction. L'article montre comment ces filières s'appuient sur plusieurs éléments (décrets d'AOC notamment) pour, finalement, maîtriser les volumes de production, donc mieux contrôler les marchés et, au final, maintenir la rentabilité de la filière. L'analyse montre aussi qu'il ne s'agit pas là de simples mesures protectionnistes mais que la recherche de la qualité est également présente dans ces pratiques collectives.

Food processing production supply a trade submitted to a competition, but also imperfectly liberalized (agricultural politicies, tariff barriers...). Concerning quality products, we find this duality. Jura's guaranteed of quality cheeses, and especially comté, thus supply a totally competitive trade but also represent a profitable, thus attractive market. Therefore there exists a genuine risk of glutting of those markets, through enterprises coming from the outside, and which would invest there, at the risk of creating overproduction. The article shows how those channels lean on several elements (especially guarantee of quality decrees) so as to, at last, master the volumes of production, so better control the markets and, finally, maintain the profitability of the channel. The analysis also shows that it is not a matter of simple protectionist measures but that the search for quality is also present in these collective practices.

\section{INDEX}

Index géographique: Jura, France

Mots-clés : fromage, politique agricole, AOC

Keywords : cheese, market, agricultural policies, PDO

\section{AUTEUR}

\section{DANIEL RICARD}

CERAMAC (EA 997), Université Blaise-Pascal, Maison des Sciences de l'Homme, 4 rue Ledru - 63

057 Clermont-Ferrand Cedex 1, France, ceramac@univ-bpclermont.fr 